\title{
Biomarcadores en fluídos biológicos y su potencial uso como indicadores de nefritis lúpica en individuos con lupus eritematoso sistémico
}

\author{
Lisandro Pacheco-Lugo ${ }^{1}$, Yirys Díaz-Olmos ${ }^{2}$, Gustavo Aroca-Martínez ${ }^{3}$ \\ 1 Investigador Grupo de Nefrología, Facultad de Medicina, Universidad Simón Bolívar, Barranquilla, Colombia. \\ 2 Estudiante de Maestría, Universidad Simón Bolívar, Barranquilla, Colombia. \\ 3 Director Científico, Clínica de la Costa. Investigador, Facultad de Medicina, Universidad Simón Bolivar, Barranquilla, Colombia.
}

\begin{abstract}
Resumen
La nefritis lúpica es una de las manifestaciones más severas del lupus eritematoso sistémico. El compromiso renal en pacientes con lupus eritematoso sistémico es un causal importante de morbilidad y mortalidad. La patogénesis de la nefritis lúpica involucra múltiples factores, entre los que se incluyen predisposición genética, regulación epigenética e interacción ambiental. Los parámetros clínicos convencionales tales como eliminación de creatinina, proteinuria, sedimentos urinarios, anticuerpos anti-ADN de doble cadena y niveles del complemento no son lo suficientemente sensibles o específicos para detectar actividad de la enfermedad. En las últimas décadas, las técnicas basadas en "Ómicas" (proteómica, genómica, transcriptómica, metabolómica) han sido utilizadas de manera extensa para la búsqueda de biomarcadores, las cuales han permitido descubrir una amplia variedad de variantes que son asociadas con lupus eritematoso sistémico y nefritis lúpica. Esos descubrimientos han expandido nuestro entendimiento de las bases moleculares de la enfermedad y han sido muy importantes para la identificación de potenciales blancos terapéuticos para predicción de la enfermedad y tratamiento temprano. En esta revisión, resumimos algunos de los estudios recientes enfocados en la identificación de biomarcadores asociados a nefritis lúpica en diversos fluidos biológicos.
\end{abstract}

Palabras clave: Nefritis lúpica, lupus eritematoso sistémico, biomarcadores, compromiso renal.

\section{Biomarkers in biological fluids and their potential use as indicators of lupus nephritis in individuals with systemic lupus erythematous}

\begin{abstract}
Abstrac
Lupus nephritis is one of the most severe manifestations of systemic lupus erythematous. Renal involvement in patients with systemic lupus ery thematous is an important cause of morbidity and mortality. The pathogenesis of lupus nephritis involve multiple factors, wich include genetic predisposition, epigenetic regulation and environmental interaction. Conventional clinical parameters such as creatinine clearance, proteinuria, urinary sediments, antibodies anti-double-strand DNA and complement proteins they are not enough sensitive or specific to detect disease activity. In the last decades, "Omics" technologies (Proteomic, genomic, transcriptomic, metobolomic) have been used in an extensive way looking for biomarkers, which allowed to discovery variants associated with systemic lupus erythematous and lupus nephritis. Such findings have expanded our knowledge about molecular basis of disease and they have been very important to identification of potential therapeutic targets to prediction of disease and early treatment. In this review, we resume some of recent studies focused in identification of biomarkers associated with lupus nephritis in diverse biological fluids. Key words: Lupus nephritis, systemic lupus erythematous, biomarkers, renal involvement.
\end{abstract}




\section{Introducción}

$\mathrm{E}$ 1 lupus eritematoso sistémico (LES), es una enfermedad crónica, autoinmune, que se caracteriza por la producción de autoanticuerpos contra un amplio rango de autoantígenos, incluyendo: ADN, ARN, histonas y otros componentes nucleares. En la mayoría de los pacientes, órganos vitales y tejidos están implicados, entre éstos el riñón, cerebro, el sistema cardiovascular, articulaciones y la piel. La nefritis lúpica (NL) es una complicación seria y muy común entre individuos que padecen LES y está asociada con tasas de morbilidad y mortalidad significativas en pacientes con LES. Aproximadamente, el $74 \%$ de los pacientes con lupus desarrollarán NL en algún momento durante el curso de su enfermedad ${ }^{1,2}$.

La patógenesis de la NL es un proceso complejo, que involucra la deposición de autoanticuerpos en el glomérulo, activación del complemento y macrófagos, proliferación celular, producción de citocinas y quimiocinas proinflamatorias, que luego se interconectan a través de múltiples mecanismos para causar daño tubular, inflamación tubulointersticial y fibrosis. Por todo esto, la NL continúa siendo una de las manifestaciones más severas del $\mathrm{LES}^{3}$. La terapia médica de la NL depende de la severidad de la enfermedad. De ésta forma, identificar biomarcadores confiables para la NL ayudará a evaluar la actividad de la enfermedad, identificar pacientes en riesgo para daño renal y facilitar el diagnóstico temprano e intervención que redunde en resultados favorables ${ }^{1,4}$.

Los actuales marcadores de laboratorio para NL tales como proteinuria, relación proteína urinaria-creatinina, eliminación de creatinina, anticuerpos anti-ADN de doble cadena, niveles del complemento, anticuerpos anti-C1q, no son lo suficientemente confiables. Todos ellos carecen de sensibilidad y especificidad para diferenciar actividad renal y daño en pacientes con NL (Tabla 1). El daño renal puede ocurrir antes de que la función renal se vea afectada y mucho antes de que se pueda detectar por parámetros clínicos de laboratorio. La proteinuria persistente no necesariamente indica inflamación en los riñones y puede obedecer a lesiones crónicas preexistentes o daño renal reciente durante el curso de la enfermedad.

\begin{tabular}{|l|c|c|}
\hline \multicolumn{3}{|c|}{ Tabla 1 } \\
\hline \multicolumn{3}{|c|}{$\begin{array}{c}\text { Rendimiento de biomarcadores históricamente } \\
\text { utilizados para predecir actividad renal lúpica }\end{array}{ }^{5-7}$. } \\
\hline Prueba & Sensibilidad & Especificidad $^{|c|}$ \\
\hline Anti-ADN de doble cadena & $53-100 \%$ & $50-69 \%$ \\
\hline C3 & $56-79 \%$ & $51-64 \%$ \\
\hline C4 & $53-74 \%$ & $64-65 \%$ \\
Anti-C1q & $53-81 \%$ & $64-71 \%$ \\
\hline \multicolumn{3}{|c|}{ Fuente: Adaptado de Reyes-Thomas, et al } \\
\hline
\end{tabular}

Las recaídas en pacientes con NL pueden ocurrir sin ningún aumento observable y reciente en el grado de proteinuria. La biopsia renal es el estándar de oro para proporcionar información de las clases histológicas de NL y el grado relativo de actividad y cronicidad en el glomérulo. Sin embargo, este procedimiento es invasivo y las biopsias seriales son poco prácticas para monitorear la NL. De esta forma, nuevos biomarcadores que sean capaces de discriminar actividad renal lúpica y su severidad, predecir recaídas renales, y monitorear la respuesta al tratamiento y el progreso de la enfermedad, se necesitan de manera urgente. En esta revisión mostramos los recientes avances y descubrimientos de nuevas moléculas que, potencialmente, podrían servir como biomarcadores del grado de compromiso renal en pacientes con nefritis lúpica.

\section{El biomarcador ideal}

Los biomarcadores se definen como eventos biológicos, bioquímicos o genéticos cuyas alteraciones se correlacionan con patogénesis o manifestaciones de la enfermedad, y que pueden ser evaluados cualitativa o cuantitativamente en laboratorios. El biomarcador ideal debería: 1) ser biológicamente activo y patofisiológicamente relevante, 2) ser simple de usar en la práctica rutinaria, y 3) cambiar sensiblemente y con precisión con la actividad de la enfermedad ${ }^{9,10}$. Adicionalmente, y no menos importante, los biomarcadores deben ser costo-efectivos, incluyendo aquellos que se utilizan en estudios de seguimiento (follow-up tests). En NL, los biomarcadores deben 
identificar pacientes en riesgo de recaídas de tal forma que la terapia pueda ser adaptada a situaciones individuales y la duración del tratamiento pueda ser determinada con precisión ${ }^{11}$.

\section{Biomarcadores séricos para nefritis lúpica}

Algunos marcadores séricos de LES incluyen: anticuerpos anti-ADN de doble cadena (anti-ADNdc), anticuerpos anti-C1q y niveles circulantes de factores del complemento como son $\mathrm{C} 3$ y C4. Éstos, sin embargo, son útiles para el diagnóstico pero son limitados a la hora de monitorear la actividad renal ${ }^{11}$. Los nucleosomas juegan un papel importante en el desarrollo de lesiones renales interviniendo en la producción y unión de autoanticuerpos a membranas basales. Un meta-análisis reveló que anticuerpos anti-nucleosoma son un marcador diagnóstico bastante exacto tanto para LES como para $\mathrm{NL}^{12}$. Adicionalmente, los anticuerpos anti-C1q séricos son también marcadores no invasivos valiosos para predecir histopatología renal en pacientes con NL. De hecho, los niveles de anticuerpos anti-C1q séricos pueden ser usados como un marcador para actividad renal con más alta sensibilidad y especificidad que marcadores tradicionales de actividad renal como es el consumo C3/C4 y anti-ADNdc ${ }^{13}$.

Panda et al., reportaron que niveles altos e intermedios de lectina de unión a manosa se asocian significativamente con NL en pacientes con $\operatorname{LES}^{14}$. Adicionalmente, se ha demostrado que los niveles del factor $\mathrm{H}$ del complemento en suero se asocian también con actividad renal en pacientes con $\mathrm{NL}^{15}$.

Análisis de la enfermedad a gran escala usando microarreglos de autoantígenos en formatos de alto rendimiento han facilitado el descubrimiento de biomarcadores de la enfermedad a escala global. Usando un arreglo proteómico glomerular, Li et al., identificaron "clusters" de autoanticuerpos que predicen de manera más sensible LES y NL activos ${ }^{16}$. Algunos de esos "clusters" de autoanticuerpos, tales como anti-cromatina, anti-ADN, anti-RO y anti-RNP, se asociaron con actividad de la enfermedad nefrítica. Estos investigadores también encontraron que la presencia de autoanticuerpos contra la inmu- noglobulina $\mathrm{M}$ en suero de pacientes se asoció con reducida severidad de $\mathrm{NL}^{17}$. Algunos estudios han sugerido que anticuerpos anti-P ribosomal en asocio con anticuerpos anti-ADNdc de doble cadena se correlacionan con una actividad nefrítica mucho mayor en pacientes con $\mathrm{LES}^{18}$. No obstante, nuestro grupo ha mostrado que los anticuerpos anti-P ribosomal no están asociados con nefritis lúpica en pacientes que sufren de LES $^{19}$.

\section{MicroARN's circulantes en suero, plasma y orina}

Los microARN's (miRNA's) son cortas secuencias de ARN no codificantes de aproximadamente $20 \mathrm{nu}$ cleótidos de longitud que regulan la expresión génica postranscripcionalmente uniéndose a regiones 3 ' no traducidas, secuencias codificantes o regiones $5^{\text {' }}$ no traducidas de ARN's mensajeros diana, llevando a la inhibición de la traducción o a la degradación del ARN mensajero. Se ha predicho que los miRNA's regulan aproximadamente el 30\% del genoma humano que codifica para proteínas ${ }^{20}$. Los miRNA's controlan la expresión de genes involucrados en varios procesos biológicos tales como apoptosis, proliferación, diferenciación y metástasis. La variación en los niveles de miRNA's podría causar la desregulación de un amplio rango de genes de blanco, los cuales están asociados con enfermedad. Los miRNA's circulantes en fluidos biológicos tienen muchas ventajas a la hora de evaluar su potencial uso como bioarcadores: los miRNA's son estables en diferentes fluidos biológicos, las secuencias de la mayoría de los miRNA's son conservados entre diferentes especies, la expresión de algunos miRNA's es tejido-específica, y el nivel de miRNA's puede ser fácilmente evaluado por varios métodos, incluyendo reacción en cadena de la polimerasa (RCP), la cual es una metodología que es utilizada de manera rutinaria en laboratorios clínicos e incluso en entidades de salud. Cambios de los niveles de miRNA's en plasma, suero, orina y saliva se han asociado con diferentes enfermedades ${ }^{21-23}$, lo cual potencia su uso como biomarcadores de enfermedad, e incluso podrían ser útiles para monitorear la progresión de ciertas patologías. La variada expresión de miRNA’s 
en el riñón durante procesos patológicos convierte a los miRNA's en una nueva herramienta valiosa para entender, diagnosticar y descubrir opciones terapéuticas para LES y nefritis lúpica. En el caso de la NL, varios estudios han mostrado una relación entre miRNA's circulantes y nefritis lúpica. La tabla 2 muestra algunos miRNA's que han sido asociados como biomarcadores de nefritis lúpica.

\section{Citocinas y quimiocinas y su papel en nefritis lúpica}

\section{Citocinas}

La NL es una manifestación clínica importante del LES. Aunque numerosas anormalidades del sistema inmune se han propuesto, la sobre-expresión de ciertas citocinas juega un papel esencial en la patogénesis de la $\mathrm{NL}^{32}$. En la fase inicial de la enfermedad los depósitos inmunes o autoanticuerpos inducen la producción de citocinas en células renales residentes, llevando a la postre a la expresión de citocinas/ quimiocinas inflamatorias e infiltración leucocitaria y activación. Luego, los leucocitos infiltrados, tales

\begin{tabular}{|l|l|l|}
\hline \multicolumn{3}{|c|}{ Tabla 2 } \\
\hline \multicolumn{2}{|c|}{$\begin{array}{l}\text { Micro-ARN's asociados como potenciales } \\
\text { biomarcadores en pacientes con nefritis lúpica. }\end{array}$} \\
\hline $\begin{array}{l}\text { Micro-ARN } \\
\text { asociado }\end{array}$ & $\begin{array}{l}\text { Fluido biológico } \\
\text { en el que fue evaluado }\end{array}$ & \multicolumn{1}{|c|}{ Autor } \\
\hline $\begin{array}{l}\text { hsa-miR-371-5P, } \\
\text { hsa-miR-423-5P, } \\
\text { hsa-miR-638, } \\
\text { hsa-miR-1224-3P } \\
\text { and hsa-miR-663 }\end{array}$ & Sangre & Te, et al. ${ }^{24}$ \\
\hline mir-146-a & Sangre & \\
\hline miR-155 & Sangre & $\begin{array}{l}\text { Dai, et al. } \\
\text { Tang, et al. }\end{array}$ \\
\hline miR-224 & Sangre & Wang, et al. $^{27}$ \\
\hline miR-21 & Sangre & Lu, et al. $^{28}$ \\
\hline miR-125-a & Sangre & $\begin{array}{l}\text { Stagakis, } \\
\text { et al. }\end{array}$ \\
\hline miR-200 ${ }^{\text {a miR-429 }}$ & Orina & Zhao, et al $^{30}$ \\
\hline
\end{tabular}

como macrófagos y células dendríticas, secretan una variedad de citocinas y activan a las células $T$ vírgenes, sesgando el perfil de citocinas secretados hacia un perfil Th1, Th2 y/o Th17. Recientes estudios revelaron esos procesos inflamatorios en modelos animales experimentales también como en modelos humanos de NL. La intervención dirigida contra citocinas puede tener el potencial terapéutico para tratar la $\mathrm{NL}^{33}$.

Los niveles séricos de la interleucina 17 (IL-17) e IL-23 se incrementan tanto en pacientes con LES con actividad lúpica como en pacientes inactivos, mientras que los niveles de IL-22 disminuyen en pacientes con lupus activo ${ }^{34}$. Aumentos en los niveles de IL-6 e INF-alpha parecen asociarse con enfermedad renal activa. Así mismo, los niveles elevados de IL-2Ra se asocian con nefritis lúpica severa y este biomarcador puede ser muy útil si se confirman estos hallazgos ${ }^{35}$. Los niveles séricos del receptor de IL-7 soluble se aumentan de manera notable en pacientes con NL, convirtiendo a esta molécula en un buen biomarcador de LES, especialmente en $\mathrm{NL}^{36}$.

\section{Quimiocinas}

Fu et al., midieron quimiocinas inducibles por interferón (RANTES, CXCL-11, MIG, IP-10, CCL-19, MCP-1) e IL-8 como biomarcadores de enfermedad activa. Los niveles de estas quimiocinas mostraron ser más altos en pacientes con LES comparados con controles saludables y controles con artritis reumatoide. CXCL-13 parece mostrar un patrón disminuido en pacientes con LES, aunque los pacientes con NL muestran un comportamiento diferente, con CXCL-13 aumentado ${ }^{37}$.

De las moléculas arriba mencionadas, la mejor estudiada es MCP-1. Hay una evidencia creciente de que MCP-1 juega un papel importante en la progresión de la falla renal basado en diferentes modelos murinos y en varias etapas de NL proliferativa ${ }^{38}$. MCP-1 es sintetizada principalmente por células mesangliales y excretada en la orina. Por lo tanto, MCP-1 en orina es un biomarcador promisorio de actividad de $\mathrm{NL}^{39}$. Además, de sus propiedades de quimioatrayente y de liberación en células mononucleares, MCP-1 parece jugar un papel importante in 
situ induciendo a la producción de citocinas proinflamatorias tales como IL-6 e ICAM-1 por parte de las células tubulares renales y mesangliales ${ }^{40}$.

Recientemente Sánchez-Muñoz et al., sugirieron que Vanina 1 podría ser un potencial biomarcador de nefritis activa en individuos con LES. Vanina 1 pertenece a una nueva ruta enzimática involucrada en inflamación, estrés oxidativo y migración celular vía cisteamina. Esta proteína tiene amplia expresión tisular pero su pico máximo de expresión es en el epitelio tisular y leucocitos periféricos. Los niveles de Vanina 1 son útiles para censar injuria renal aguda inducida por solventes orgánicos, drogas y diabetes $^{41}$. Utilizando PCR en tiempo real y ELISA, estos investigadores encontraron niveles significativamente elevados en sangre y orina de Vanina 1 en pacientes con LES, con actividad renal, comparados con pacientes con artritis reumatoide, sin afectación renal. No obstante, en este estudio solo se evaluaron 20 pacientes con LES, 7 de los cuales presentaban actividad renal, lo que no permite hacer un análisis de los resultados de manera exhaustiva. Es necesario replicar este estudio utilizando una muestra mucho más grande.

\section{Biomarcadores urinarios}

Generalmente, las sustancias urinarias reflejan daño renal mejor que componentes en el suero. La orina es una fuente de biofluídos que es fácil de colectar y los biomarcadores en orina, usualmente, reflejan la función renal directamente en varios tipos de enfermedad nefrítica. Abordes proteómicos, tales como electroforesis bidimensional y espectrometría de masas, han sido ampliamente utilizados para analizar potenciales biomarcadores urinarios que están asociados con daño renal causado por NL. ET-1, por ejemplo, es un péptido de 21 aminoácidos producido en la vasculatura que participa en la proliferación celular, inflamación, vasoconstricción y fibrosis. Los niveles de la fracción de excreción de ET-1 muestran un aumento significativo durante la progresión tanto de enfermedad renal crónica como de NL. Los niveles de esta molécula disminuyeron después de terapia en pacientes con NL, de tal forma que este biomarcador puede ser de gran utilidad ${ }^{42}$.

\section{Lipocalina-2}

Lipocalina-2, secretada por leucocitos y células epiteliales, es importante para el transporte de hierro. Los niveles urinarios de esta molécula se evaluaron en 70 pacientes con LES (con o sin nefritis) y en controles saludables. Los niveles en orina de lipocalina-2 fueron predictivos de nefritis activa ${ }^{43}$.

\section{VCAM-1}

Wu et al., compararon las muestras de orina de 38 pacientes con LES vs. 15 controles normales y 6 pacientes control con artritis reumatoide, y encontraron que los pacientes con LES con nefritis activa tenían más altos niveles de VCAM-1 en orina que los controles $^{44}$. Resultados similares fueron reportados por Kiani et al., quienes encontraron que los niveles de VCAM-1 urinario se correlacionaron fuertemente con una actividad renal más alta, hematuria, proteinuria y piuria en 81 pacientes con $\mathrm{LES}^{45}$.

\section{IL-6}

Los niveles urinarios de la IL-6 también han sido evaluados como potenciales biomarcadores de daño renal en pacientes con LES. Los niveles de IL-6 en orina se evaluaron en 29 pacientes con NL activa, encontrándose que pacientes con NL grado IV tenían niveles más altos de esta interleucina que pacientes con otra clase de nefritis ${ }^{46,47}$.

\section{IP-10}

La proteína 10 inducible por interferón gamma (IP-10), también conocida como CXCL10, es una quimiocina secretada por células endoteliales estimuladas por interferón gamma, fibroblastos y monocitos. Junto con su receptor CXCR3, IP-10 promueve la migración de células $\mathrm{T}$ a sitios de inflamación y también se conoce que juega un papel en la regulación negativa de angiogénesis. Dada la relevancia patogénica conocida de IP-10 y los niveles incrementados encontrados en el suero de pacientes con LES, Avihingsanon et al., estudiaron 26 pacientes, 14 de los cuales tenían NL clase IV, 12 de ellos tenían NL clase II, III, V o VI. Ellos 
examinaros los niveles de ARNm urinario de IP-10, CXCR3, factor de crecimiento transformante beta (TGF-b) y el factor de crecimiento endotelial vascular, y encontraron que los niveles de todos los 4 ARNm estuvieron incrementados en pacientes con nefritis lúpica clase IV cuando se comparó con las otras clases de nefritis. De manera interesante, los pacientes que respondieron a la terapia tenían significativos niveles más bajos de IP-10, sugiriendo que IP-10 puede ser usado como un barómetro para eficacia de tratamiento ${ }^{48}$.

\section{TWEAK}

La citocina TWEAK fue descubierta en $1997^{49}$. Se asignó como miembro de la familia del factor de necrosis tumoral alfa (TNF-a) basado en motivos de secuencias. En las células del riñón TWEAK interviene en importantes sucesos biológicos, incluyendo modulación de la supervivencia celular y sobrerregulación de mediadores proinflamatorios ${ }^{50}$.

Schwartz et al., examinaron los niveles urinarios de TWEAK en un grupo de estudio de pacientes con lupus. Ellos encontraron que los niveles de TWEAK estuvieron significativamente más altos en pacientes con nefritis activa, comprados con pacientes con nefritis inactiva ${ }^{51}$. Ellos también encontraron una correlación significativa entre TWEAK urinario y conteo SLEDAI, indicando una relación linear entre los niveles de TWEAK y el umbral de actividad nefrítica. El mismo grupo reportó resultados similares en otra cohorte de pacientes con LES ${ }^{52}$.

Suzuki et al., utilizando espectrometría de masas, identificaron una serie de proteínas aumentadas en la orina de niños con NL comparados con individuos sin actividad renal. Las proteínas identificadas fueron: transferrina, glicoproteína ácida alfa 1, prostaglandina-D sintetasa tipo lipocalina, albúmina y fragmentos relacionados a albúmina ${ }^{53}$. Estos resultados fueron confirmados por Brunner et al., en un estudio posterior ${ }^{54}$. Ceruloplasmina o como es conocida oficialmente: ferroxidasa, es la principal proteína transportadora de cobre en la sangre. Esta proteína fue encontrada expresada en mayor nivel en individuos con NL activa comparados con pacientes con LES sin nefritis ${ }^{54}$.

\section{Conclusiones}

El actual tratamiento de la NL severa es insatisfactorio en términos de resolución de la enfermedad y toxicidad. Para mejorar la eficacia y disminución de los efectos adversos de la inmunosupresión, sería ideal poder predecir el curso y la patología de la NL y ajustar la terapia de manera apropiada. Esto requerirá biomarcadores que reflejen la actividad de la enfermedad. Recientemente, un esfuerzo significativo ha sido puesto en tratar de identificar biomarcadores circulantes que puedan anticipar recaídas renales en pacientes con NL.

El muestreo de moléculas revisadas en este artículo ejemplifica el interés creciente de encontrar un verdadero biomarcador para la NL. Aunque muchos de los estudios son preliminares, los resultados son bastante motivadores. Con los recientes avances a nivel proteómico y las tecnologías de ultrasecuenciación cada vez más robustas, hay un optimismo en que pronto un biomarcador emergerá con la posibilidad de contribuir al manejo de la enfermedad y disminuir las altas tasas de morbilidad y mortalidad. No obstante, en la actualidad nadie ha evaluado estos potenciales biomarcadores en grandes cohortes longitudinales de pacientes. En este sentido, quizá sería mucho mejor destinar esfuerzos hacia estudios clínicos que determinen el poder predictivo de esas moléculas conocidas en grandes estudios multicéntricos con pacientes con LES, en vez de seguir buscando nuevas moléculas. Solo entonces descubriremos si esos promisorios biomarcadores constituyen una esperanza para mejorar la calidad de vida de pacientes con $\mathrm{LES}^{8}$.

En nuestra opinión, es probable que la combinación de perfiles de biomarcadores, más que biomarcadores individuales, emerja para ayudar a predecir la severidad de la inflamación, el nivel de fibrosis, el grado de respuesta a drogas y otras variables. Este aborde tiene el potencial de limitar el uso de la biopsia renal. De manera adicional, esto debería mejorar la eficacia terapéutica y limitar la toxicidad.

IP-10 como biomarcador promete en su capacidad diagnóstica ya que parece ser bastante específico para pacientes con NL clase IV y, si esta información se valida en otros estudios con cohortes de pacientes 
mucho más grandes, IP-10 podría ser potencialmente usada en lugar de la biopsia renal para ordenar tratamiento o cambio en el manejo de la enfermedad. Indudablemente, se necesitan más estudios que validen su potencial uso como biomarcador candidato ${ }^{8}$.

\section{Conflicto de interés}

Los autores declaran no presentar ningún conflicto de interés.

\author{
Abreviaturas \\ LES, Lupus eritematoso sistémico. \\ NL, Nefritis lúpica.
}

ADN, Ácido desoxirribonucleico

ADNdc, Ácido desoxirribonucleico de doble cadena ARN, Ácido ribonucleico

RNP, Ribonucleoproteína

miRNA's, Micro-ARN's

RCP, Reacción en cadena de la polimerasa

IL, Interleucinas

ELISA, Ensayo inmunoabsorbente ligado a enzima

VCAM, Proteína de adhesión de células vasculares

ICAM, Molécula de adhesión intercelular

TWEAK, Citocina débil inductora de apoptosis de la familia del factor de necrosis tumoral

\section{Referencias bibliográficas}

1. Li, Y., Fang, X. \& Li, Q.-Z. Biomarker profiling for lupus nephritis. Genomics Proteomics Bioinformatics 2013;11,158-165.

2. Cervera, R. et al. Systemic lupus erythematosus in Europe at the change of the millennium: lessons from the 'Euro-Lupus Project'. Autoimmun. Rev. 2006;5,180-186.

3. Gurevitz, S. L., Snyder, J. A., Wessel, E. K., Frey, J. \& Williamson, B. A. Systemic lupus erythematosus: a review of the disease and treatment options. Consult. Pharm. J. Am. Soc. Consult. Pharm. 2013;28,110-121.

4. Liu, C.-C., Manzi, S. \& Ahearn, J. M. Biomarkers for systemic lupus erythematosus: a review and perspective. Curr. Opin. Rheumatol. 2005;17,543-549.

5. Oelzner, P. et al. Anti-C1q antibodies and antiendothelial cell antibodies in systemic lupus erythematosus - relationship with disease activity and renal involvement. Clin. Rheumatol. 2003;22,271-278.

6. Moroni, G. et al. Are laboratory tests useful for monitoring the activity of lupus nephritis? A 6-year prospective study in a cohort of 228 patients with lupus nephritis. Ann. Rheum. Dis. 2009;68,234-237.

7. Esdaile, J. M. et al. Routine immunologic tests in systemic lupus erythematosus: is there a need for more studies? J. Rheumatol. 1996;23,1891-1896.

8. Reyes-Thomas, J., Blanco, I. \& Putterman, C. Urinary Biomarkers in Lupus Nephritis. Clin. Rev. Allergy Immunol. $2011 ; 40,138-150$.

9. Illei, G. G. \& Lipsky, P. E. Biomarkers in systemic lupus erythematosus. Curr. Rheumatol. Rep. 2004;6,382-390.

10. Illei, G. G., Tackey, E., Lapteva, L. \& Lipsky, P. E. Biomarkers in systemic lupus erythematosus: II. Markers of disease activity. Arthritis Rheum. 2004;50,2048-2065.

11. Manoharan, A. \& Madaio, M. P. Biomarkers in Lupus Nephritis. Rheum. Dis. Clin. N. Am. 2010;36,131-143.

12. Simón, J. A., Cabiedes, J., Ortiz, E., Alcocer-Varela, J. \& Sánchez-Guerrero, J. Anti-nucleosome antibodies in patients with systemic lupus erythematosus of recent onset. Potential utility as a diagnostic tool and disease activity marker. Rheumatol. Oxf. Engl. 2004;43,220-224. 
13. Marto, N., Bertolaccini, M. L., Calabuig, E., Hughes, G. R. V. \& Khamashta, M. A. Anti-C1q antibodies in nephritis: correlation between titres and renal disease activity and positive predictive value in systemic lupus erythematosus. Ann. Rheum. Dis. 2005;64,444-448.

14. Panda, A. K. et al. Mannose binding lectin: a biomarker of systemic lupus erythematosus disease activity. Arthritis Res. Ther. 2012;14,R218.

15. Wang, F., Yu, F., Tan, Y., Song, D. \& Zhao, M. Serum complement factor H is associated with clinical and pathological activities of patients with lupus nephritis. Rheumatol. Oxf. Engl. 2012;51,2269-2277.

16. Li, Q.-Z. et al. Identification of autoantibody clusters that best predict lupus disease activity using glomerular proteome arrays. J. Clin. Invest. 2005;115,3428-3439.

17. Li, Q.-Z. et al. Protein array autoantibody profiles for insights into systemic lupus erythematosus and incomplete lupus syndromes. Clin. Exp. Immunol. 2007;147,60-70.

18. Egner, W. The use of laboratory tests in the diagnosis of SLE. J. Clin. Pathol. 2000;53,424-432.

19. Quintana, G. et al. Single anti-P ribosomal antibodies are not associated with lupus nephritis in patients suffering from active systemic lupus erythematosus. Autoimmun. Rev. 2010;9,750-755.

20. Filipowicz, W., Bhattacharyya, S. N. \& Sonenberg, N. Mechanisms of post-transcriptional regulation by microRNAs: are the answers in sight? Nat. Rev. Genet. 2008;9,102-114.

21. Mitchell, P. S. et al. Circulating microRNAs as stable blood-based markers for cancer detection. Proc. Natl. Acad. Sci. U. S. A. 2008;105,10513-10518.

22. Zhao, H. et al. A pilot study of circulating miRNAs as potential biomarkers of early stage breast cancer. PloS One 2010;5,e13735.

23. Roth, C. et al. Circulating microRNAs as blood-based markers for patients with primary and metastatic breast cancer. Breast Cancer Res. BCR 2010;12,R90.

24. Te, J. L. et al. Identification of Unique MicroRNA Signature Associated with Lupus Nephritis. PLoS ONE 2010;5,e10344.

25. Dai, Y. et al. Microarray analysis of microRNA expression in peripheral blood cells of systemic lupus erythematosus patients. Lupus 2007;16,939-946.

26. Tang, Y. et al. MicroRNA-146A contributes to abnormal activation of the type I interferon pathway in human lupus by targeting the key signaling proteins. Arthritis Rheum. 2009;60,1065-1075.

27. Wang, G. et al. Serum and urinary cell-free MiR-146a and MiR-155 in patients with systemic lupus erythematosus. J. Rheumatol. 2010;37,2516-2522.

28. Lu, M.-C. et al. Decreased microRNA(miR)-145 and increased miR-224 expression in T cells from patients with systemic lupus erythematosus involved in lupus immunopathogenesis. Clin. Exp. Immunol. 2013;171,91-99.

29. Stagakis, E. et al. Identification of novel microRNA signatures linked to human lupus disease activity and pathogenesis: miR-21 regulates aberrant T cell responses through regulation of PDCD4 expression. Ann. Rheum. Dis. 2011;70,1496-1506.

30. Zhao, X. et al. MicroRNA-125a contributes to elevated inflammatory chemokine RANTES levels via targeting KLF13 in systemic lupus erythematosus. Arthritis Rheum. 2010;62,3425-3435.

31. Wang, G. et al. Serum and urinary free microRNA level in patients with systemic lupus erythematosus. Lupus 2011;20,493-500.

32. Gómez-Guerrero, C., Hernández-Vargas, P., López-Franco, O., Ortiz-Muñoz, G. \& Egido, J. Mesangial cells and glomerular inflammation: from the pathogenesis to novel therapeutic approaches. Curr. Drug Targets Inflamm. Allergy $2005 ; 4,341-351$.

33. Adhya, Z., Borozdenkova, S. \& Karim, M. Y. The role of cytokines as biomarkers in systemic lupus erythematosus and lupus nephritis. Nephrol. Dial. Transplant. Off. Publ. Eur. Dial. Transpl. Assoc. - Eur. Ren. Assoc. 2011;26,32733280 .

Biomarcadores en fluídos biológicos y su potencial uso como indicadores de nefritis lúpica en individuos con lupus eritematoso sistémico 
34. Sabry, A. et al. Proinflammatory cytokines (TNF-alpha and IL-6) in Egyptian patients with SLE: its correlation with disease activity. Cytokine 2006;35,148-153.

35. El-Shafey, E. M., El-Nagar, G. F., El-Bendary, A. S., Sabry, A. A. \& Selim, A.-G. A. Serum soluble interleukin-2 receptor alpha in systemic lupus erythematosus. Iran. J. Kidney Dis. 2008;2,80-85.

36. Badot, V. et al. Serum soluble interleukin 7 receptor is strongly associated with lupus nephritis in patients with systemic lupus erythematosus. Ann. Rheum. Dis. 2013;72,453-456.

37. Fu, Q. et al. Association of elevated transcript levels of interferon-inducible chemokines with disease activity and organ damage in systemic lupus erythematosus patients. Arthritis Res. Ther. 2008;10,R112.

38. Segerer, S., Nelson, P. J. \& Schlöndorff, D. Chemokines, chemokine receptors, and renal disease: from basic science to pathophysiologic and therapeutic studies. J. Am. Soc. Nephrol. JASN 2000;11,152-176.

39. Alzawawy, A., Zohary, M., Ablordiny, M. \& Eldalie, M. Estimation of monocyte-chemoattractantprotein-1 (Mcp-1) level in patients with lupus nephritis. Int. J. Rheum. Dis. 2009;12,311-318.

40. Viedt, C. \& Orth, S. R. Monocyte chemoattractant protein-1 (MCP-1) in the kidney: does it more than simply attract monocytes? Nephrol. Dial. Transplant. Off. Publ. Eur. Dial. Transpl. Assoc. - Eur. Ren. Assoc. 2002;17,2043-2047.

41. Sánchez-Muñoz, F., Amezcua-Guerra, L., Macías-Palacios, M., Márquez-Velasco, R. \& Bojalil, R. Vanin-1 as a potential novel biomarker for active nephritis in systemic lupus erythematosus. Lupus 2013;22,333-335.

42. Dhaun, N. et al. Urinary endothelin-1 in chronic kidney disease and as a marker of disease activity in lupus nephritis. Am. J. Physiol. Renal Physiol. 2009;296,F1477-1483.

43. Pitashny, M. et al. Urinary lipocalin-2 is associated with renal disease activity in human lupus nephritis. Arthritis Rheum. 2007;56,1894-1903.

44. Wu, T. et al. Elevated urinary VCAM-1, P-selectin, soluble TNF receptor-1, and CXC chemokine ligand 16 in multiple murine lupus strains and human lupus nephritis. J. Immunol. Baltim. Md 1950 2007;179,7166-7175.

45. Kiani, A. N. et al. Urinary vascular cell adhesion molecule, but not neutrophil gelatinase-associated lipocalin, is associated with lupus nephritis. J. Rheumatol. 2012;39,1231-1237.

46. Iwano, M. et al. Urinary levels of IL-6 in patients with active lupus nephritis. Clin. Nephrol. 1993;40,16-21.

47. Tsai, C. Y., Wu, T. H., Yu, C. L., Lu, J. Y. \& Tsai, Y. Y. Increased excretions of beta2-microglobulin, IL-6, and IL-8 and decreased excretion of Tamm-Horsfall glycoprotein in urine of patients with active lupus nephritis. Nephron $2000 ; 85,207-214$.

48. Avihingsanon, Y. et al. Measurement of urinary chemokine and growth factor messenger RNAs: a noninvasive monitoring in lupus nephritis. Kidney Int. 2006;69,747-753.

49. Chicheportiche, Y. et al. TWEAK, a new secreted ligand in the tumor necrosis factor family that weakly induces apoptosis. J. Biol. Chem. 1997;272,32401-32410.

50. Campbell, S., Michaelson, J., Burkly, L. \& Putterman, C. The role of TWEAK/Fn14 in the pathogenesis of inflammation and systemic autoimmunity. Front. Biosci. J. Virtual Libr. 2004;9,2273-2284.

51. Schwartz, N. et al. Urinary TWEAK and the activity of lupus nephritis. J. Autoimmun. 2006;27,242-250.

52. Schwartz, N. et al. Urinary TWEAK as a biomarker of lupus nephritis: a multicenter cohort study. Arthritis Res. Ther. 2009;11,R143.

53. Suzuki, M. et al. Initial Validation of a Novel Protein Biomarker Panel for Active Pediatric Lupus Nephritis. Pediatr. Res. 2009;65,530-536.

54. Brunner, H. I. et al. Association of noninvasively measured renal protein biomarkers with histologic features of lupus nephritis. Arthritis Rheum. 2012;64,2687-2697. 